\title{
Spectrum Estimation, Notch Filters, and MUSIC
}

\author{
Kaushik Mahata
}

\begin{abstract}
A novel extension of the Multiple Signal Classification (MUSIC) algorithm for the frequency estimation problem is proposed in this work. It is shown that the MUSIC algorithm is a data-dependent optimum notch filter design technique. When the input data are passed through this optimum notch filter, the signal-to-noise ratio (SNR) of the output is minimized. The zeros of the optimum notch filter are then used to estimate the frequencies. In the classical MUSIC algorithm, this optimization is carried out over the set of all finite impulse response (FIR) filters having a fixed order. The framework of this paper allows the use of rational notch filters. The fact that the rational filters have sharper spectral characteristics is used to obtain improved estimates, particularly at low SNR. Some interesting properties of the proposed algorithm are derived, and further perspectives on the implementation aspects are provided. The analytical predictions are substantiated using numerical simulation results.
\end{abstract}

Index Terms-Frequency estimation, MUSIC, rational notch filters, subspace algorithms.

\section{INTRODUCTION}

$\mathbf{C}$ ONSIDER that we observe a complex-valued data sequence $y(t)$ comprised of sinusoids in noise:

$$
y_{0}(t)=\sum_{k=1}^{d} c_{k} e^{i\left(\omega_{k} t+\phi_{k}\right)}, \quad y(t)=y_{0}(t)+\tilde{y}(t) .
$$

Here, the amplitudes $\left\{c_{k}\right\}_{k=1}^{d}$, frequencies $\left\{\omega_{k}\right\}_{k=1}^{d}$, and the initial phases $\left\{\phi_{k}\right\}_{k=1}^{d}$ are real-valued unknowns. The complexvalued measurement noise sequence $\{\tilde{y}(t)\}$ is assumed to be stationary, zero mean, white, and circular. The variance of the noise process $\{\tilde{y}(t)\}$ is unknown and is denoted by $\sigma^{2}$. The initial phases $\left\{\phi_{k}\right\}_{k=1}^{d}$ are mutually independent and identically distributed (i.i.d.) uniformly in $[0,2 \pi)$. Here, we are concerned about the classical problem of estimating the parameter vector of real-valued frequencies

$$
\boldsymbol{\omega}=\left[\begin{array}{lll}
\omega_{1} & \cdots & \omega_{d}
\end{array}\right]
$$

from $N$ samples of the observed data $\{y(t)\}_{t=1}^{N}$.

Subspace algorithms [1]-[5] for the frequency estimation problem have been established as accurate and computationally economical alternatives of the maximum likelihood (ML) method [5], [6]. However, the resolution capability and the estimation performance of the subspace algorithms degrade rapidly with decrease in the signal-to-noise ratio (SNR) of

Manuscript received June 16, 2004; revised November 29, 2004. This work was supported by the Australian Research Council. The associate editor coordinating the review of this paper and approving it for publication was Prof. Jian Li.

The author is with the Center for Complex Dynamic Systems and Control, School of Electrical Engineering, University of Newcastle, Callaghan, NSW2308, Australia (e-mail: kush@ee.newcastle.edu.au).

Digital Object Identifier 10.1109/TSP.2005.855395 the data. In fact, it has been shown in many related papers that the asymptotic covariance matrices of the subspace-based frequency estimates are inversely proportional to the square of the SNR [3], [7]. In finite samples, the performance deterioration is even more prominent. Hence, the problem of designing subspace-based frequency estimation techniques that can complement the ML method at low SNR is still a relevant research question. In this work, our primary objective is to address this particular issue.

We propose a novel extension of the Multiple Signal Classification (MUSIC) algorithm [1], [5], [8], [9] in this paper. MUSIC has two different versions. The first version is known as the spectral MUSIC [1], [8] algorithm. Determination of the frequencies via spectral MUSIC requires accurate localization of the multiple peaks of a one-dimensional pseudo-spectrum. This involves a nonlinear search over a one-dimensional multimodal surface. The second version of MUSIC, namely Root-MUSIC, was proposed in [9]. Root-MUSIC is based on a polynomial formulation of the above-mentioned spectral search. In this case, the frequencies are estimated by computing the angular positions of the $d$ (pairs of reciprocal) roots of an equation located nearest to the unit circle. Apart from being computationally more economical, Root-MUSIC gives superior estimation performance than spectral MUSIC [10].

Recently, a new paradigm for spectral analysis has been proposed in the framework of analytic interpolation using input-tostate filters [11]-[13]. It has been shown in [12] and [13] that the performance of many subspace methods can be improved using input-to-state filters. In this paper, we explore further properties of the MUSIC algorithm based on input-to-state filters. We show in a generalized presentation that the MUSIC algorithm can be viewed as a data-dependent optimum notch filter design technique. When the input data are passed through this optimum notch filter, the corresponding output SNR is minimized. The zeros of the optimum notch filter are then used to estimate $\boldsymbol{\omega}$. In the case of the classical MUSIC algorithm the optimization is carried out over the set of finite impulse response (FIR) filters. In the framework presented in [12] and [13], we can allow rational notch filters. The fact that rational notch filters have sharper spectral characteristics plays a crucial role in improving the estimation performance. Using the notch filtering interpretation, it is possible to provide a qualitative insight into the behavior of the extended MUSIC algorithm. This additional insight can be useful in many other applications employing similar subspace methods like array processing [5] and blind identification [14]. A novel alternative extension of MUSIC based on input-to-state filters is proposed in the sequel, and a consistency analysis thereof is carried out. The alternative algorithm needs to solve only one eigenvalue problem instead of two in [12] and [13]. Further qualitative and quantitative insights on the issues, 
like designing the input-to-state filter and the implementation aspects, are provided. Analytical predictions are also supported by numerical simulation results, where we achieve a significant performance (resolution and accuracy) gain at low SNR.

\section{NOTCH FILTERS AND MUSIC}

\section{A. Optimum Notch Filters}

In this paper, we use $q^{-1}$ to denote the unit delay operator: $q^{-1} y(t)=y(t-1)$. Let us introduce the polynomial $A\left(q^{-1}, \boldsymbol{\omega}\right)$ having $\left\{e^{i \omega_{k}}\right\}_{k=1}^{d}$ as its roots:

$$
A\left(q^{-1}, \boldsymbol{\omega}\right):=\prod_{k=1}^{d}\left(1-e^{i \omega_{k}} q^{-1}\right):=1+\sum_{k=1}^{d} a_{k}(\boldsymbol{\omega}) q^{-k}
$$

Many popular super-resolution subspace algorithms obtain an estimate of $A\left(q^{-1}, \boldsymbol{\omega}\right)$ first. Subsequently, the frequency estimates are recovered via polynomial rooting. Eigen-analysis approaches described in [3] and [4] and Pisarenko's harmonic decomposition method [15] are a few well-known examples. Note that $A\left(q^{-1}, \boldsymbol{\omega}\right)$ is an annihilating filter for the true signal $y_{0}(t)$, i.e.,

$$
A\left(q^{-1}, \boldsymbol{\omega}\right) y_{0}(t)=0
$$

after transients due to the initial conditions die down (in this case, the length of the transient is $d$ ). All such annihilating filters share a common property. They are notch filters having zeros at $\left\{e^{i \omega_{k}}\right\}_{k=1}^{d}$. Annihilating filters are not necessarily FIR. We can have rational filters as well. However, the denominator of a rational annihilating filter has no direct influence on the annihilation process. Therefore, the poles of such annihilating filters are flexible. The main idea in this section is to estimate the annihilating filter numerator (given a prespecified denominator) from the data. Subsequently, the zeros of the estimated numerator polynomial give the frequency estimates.

Suppose that the input signal $y(t)$ is passed through a stable rational filter $G\left(q^{-1}, \boldsymbol{\ell}, \boldsymbol{\delta}\right)$ of order $m(m>d)$ having the form

$$
\begin{aligned}
G\left(q^{-1}, \boldsymbol{\ell}, \boldsymbol{\delta}\right) & :=\frac{L\left(q^{-1}, \boldsymbol{\ell}\right)}{D\left(q^{-1}, \boldsymbol{\delta}\right)} \\
L\left(q^{-1}, \boldsymbol{\ell}\right) & =\sum_{k=0}^{m-1} \ell_{k} q^{-k} \\
D\left(q^{-1}, \boldsymbol{\delta}\right) & =1+\sum_{k=1}^{m} \delta_{k} q^{-k}
\end{aligned}
$$

where the parameter vectors $\boldsymbol{\ell}$ and $\boldsymbol{\delta}$ are defined as

$$
\boldsymbol{\ell}=\left[\begin{array}{lll}
\ell_{0} & \cdots & \ell_{m-1}
\end{array}\right], \quad \boldsymbol{\delta}=\left[\begin{array}{lll}
\delta_{1} & \cdots & \delta_{m}
\end{array}\right]
$$

Denote the corresponding output from $G\left(q^{-1}, \boldsymbol{\ell}, \boldsymbol{\delta}\right)$ by $y_{f}(t)$ :

$$
y_{f}(t):=G\left(q^{-1}, \boldsymbol{\ell}, \boldsymbol{\delta}\right) y(t) .
$$

Here, we fix $\delta$ [and, hence, the denominator $D\left(q^{-1}, \delta\right)$ of the rational filter $\left.G\left(q^{-1}, \boldsymbol{\ell}, \boldsymbol{\delta}\right)\right]$ and then compute a vector $\boldsymbol{\ell}$ such that $L\left(q^{-1}, \boldsymbol{\ell}\right)$ gives an estimate of the annihilating numerator. Given the noise-corrupted data, this can be achieved by minimizing the
SNR of $y_{f}(t)$. Here, the minimization is carried out with respect to $\boldsymbol{\ell}$. Let the SNR of $y_{f}(t)$ be given by $\rho(\boldsymbol{\ell}, \boldsymbol{\delta})$. Then, we denote

$$
\begin{aligned}
\boldsymbol{\ell}_{\star}(\boldsymbol{\delta}) & :=\arg \min _{\boldsymbol{\ell}} \rho(\boldsymbol{\ell}, \boldsymbol{\delta}) \\
L_{\star}\left(q^{-1}, \boldsymbol{\delta}\right) & :=L\left\{q^{-1}, \boldsymbol{\ell}_{\star}(\boldsymbol{\delta})\right\} .
\end{aligned}
$$

Note that $\boldsymbol{\ell}_{\star}(\boldsymbol{\delta})$ depends on $\boldsymbol{\delta}$. Consider the case when $m=$ $d+1$. The annihilating numerator is uniquely (up to a scale factor) given by $A\left(q^{-1}, \boldsymbol{\omega}\right)$, but when the data length $N$ is finite and the measurements are noisy, $L_{\star}\left(q^{-1}, \delta\right)$ varies with $\delta$. By forcing $L_{\star}\left(q^{-1}, \boldsymbol{\delta}\right)$ to be a monic polynomial, we get an estimate of $A\left(q^{-1}, \omega\right)$, and the statistical property of this estimator depends on $\boldsymbol{\delta}$. By introducing rational annihilating filters, we get the advantage of tuning $\delta$ to achieve improved estimation performance.

The SNR minimization problem introduced above can be solved analytically by considering a state-space realization of $G\left(q^{-1}, \boldsymbol{\ell}, \boldsymbol{\delta}\right)$. Let the filter $G\left(q^{-1}, \boldsymbol{\ell}, \boldsymbol{\delta}\right)$ be expressed in a suitable state-space form with an associated state vector $\boldsymbol{x}(t)$ :

$$
\begin{aligned}
\boldsymbol{x}(t) & =\mathbf{F} \boldsymbol{x}(t-1)+\boldsymbol{g} y(t) \\
y_{f}(t) & =\boldsymbol{h} \boldsymbol{x}(t) .
\end{aligned}
$$

Here, $\mathbf{F}$ is an $m \times m$ matrix, $\boldsymbol{h}$ is an $1 \times m$ vector, and $\boldsymbol{g}$ is an $m \times 1$ vector such that

$$
G\left(q^{-1}, \boldsymbol{\ell}, \boldsymbol{\delta}\right)=\boldsymbol{h}\left[\mathbf{I}_{m}-q^{-1} \mathbf{F}\right]^{-1} \boldsymbol{g}
$$

where $\mathbf{I}_{m}$ is the identity matrix of size $m$. To keep the notations simple, we do not show the dependence of state-space matrices $\mathbf{F}, \boldsymbol{g}$, and $\boldsymbol{h}$ on the parameters $\boldsymbol{\ell}$ and $\boldsymbol{\delta}$ explicitly. Note that $D\left(q^{-1}, \boldsymbol{\delta}\right)$ is the characteristic polynomial of $\mathbf{F}$. In what follows next, we express the SNR $\rho(\boldsymbol{\ell}, \boldsymbol{\delta})$ of $y_{f}(t)$ in terms of the covariance matrix of the state vector $\boldsymbol{x}(t)$. Subsequently, we prove that minimizing $\rho(\boldsymbol{\ell}, \boldsymbol{\delta})$ with respect to $\boldsymbol{\ell}$ is equivalent to minimizing $\rho(\boldsymbol{\ell}, \boldsymbol{\delta})$ with respect to $\boldsymbol{h}$. In this way, we can solve the optimization problem analytically.

Without any loss of generality, we assume that the stat-space realization in (7) and (8) is controllable and observable. Let the noise contribution to the state sequence $\boldsymbol{x}(t)$ be denoted by $\tilde{\boldsymbol{x}}(t)$. Define

$$
\mathbf{Q}:=\mathcal{E}\left\{\boldsymbol{x}(t) \boldsymbol{x}^{*}(t)\right\}, \quad \mathbf{E}:=\frac{1}{\sigma^{2}} \mathcal{E}\left\{\tilde{\boldsymbol{x}}(t) \tilde{\boldsymbol{x}}^{*}(t)\right\}
$$

where $\boldsymbol{x}^{*}$ denotes the conjugate transpose of $\boldsymbol{x}$, and $\mathcal{E}(\cdot)$ denotes the mathematical expectation operator. Recall that $\tilde{y}(t)$ is a white noise. Therefore, the normalized noise covariance matrix E satisfies the Lyapunov equation [12]

$$
\mathbf{E}=\mathbf{F E F}^{*}+\boldsymbol{g g}^{*} \text {. }
$$

Now, from (7) and (8), we see that the average power $\wp(\boldsymbol{\ell}, \boldsymbol{\delta})$ of $y_{f}(t)$ and the noise contribution $\tilde{\wp}(\boldsymbol{\ell}, \boldsymbol{\delta})$ therein satisfy

$$
\wp(\boldsymbol{\ell}, \boldsymbol{\delta})=\boldsymbol{h} \mathbf{Q} \boldsymbol{h}^{*}, \quad \tilde{\wp}(\boldsymbol{\ell}, \boldsymbol{\delta})=\sigma^{2} \boldsymbol{h} \mathbf{E} \boldsymbol{h}^{*} .
$$

Therefore, the SNR $\rho(\boldsymbol{\ell}, \boldsymbol{\delta})$ of $y_{f}(t)$ is given by

$$
\rho(\boldsymbol{\ell}, \boldsymbol{\delta})=\frac{\wp(\boldsymbol{\ell}, \boldsymbol{\delta})-\tilde{\wp}(\boldsymbol{\ell}, \boldsymbol{\delta})}{\tilde{\wp}(\boldsymbol{\ell}, \boldsymbol{\delta})}=\frac{\boldsymbol{h} \mathbf{Q} \boldsymbol{h}^{*}}{\sigma^{2} \boldsymbol{h} \mathbf{E} \boldsymbol{h}^{*}}-1 .
$$


We emphasize at this point that $\sigma^{2}$ being unknown is not a problem for the SNR minimization approach because minimizing $\rho(\boldsymbol{\ell}, \boldsymbol{\delta})$ is equivalent to minimizing $\left\{\boldsymbol{h} \mathbf{Q} h^{*}\right\} /\left\{\boldsymbol{h} \mathbf{E} \boldsymbol{h}^{*}\right\}$. This minimization can be carried out analytically with respect to $\boldsymbol{h}$. In the next step, we show that for a fixed controllable pair $(\mathbf{F}, \boldsymbol{g})$, there exists a bijective linear mapping between $\boldsymbol{h}$ and $\boldsymbol{\ell}$ so that (9) holds. Now note that the matrices $\mathbf{Q}$ and $\mathbf{E}$ depend only on $\mathbf{F}$ and $\boldsymbol{g}$ and are independent of $\boldsymbol{h}$. Therefore, we can first minimize $\left\{\boldsymbol{h} \mathbf{Q} h^{*}\right\} /\left\{\boldsymbol{h} \mathbf{E} \boldsymbol{h}^{*}\right\}$ with respect to $\boldsymbol{h}$ and then recover $\boldsymbol{\ell}_{\star}$ from the minimizing $\boldsymbol{h}$ via a linear transformation.

Proposition 1: Denote the $k$ th column of the $m \times m$ identity matrix $\mathbf{I}_{m}$ by $\boldsymbol{e}_{k}$, and define the $m \times m$ matrices $\mathbf{F}_{*}$ and $\mathcal{C}_{*}$ as

$$
\begin{aligned}
\mathbf{F}_{*} & =\left[\begin{array}{llll}
-\boldsymbol{\delta}^{\top} & \boldsymbol{e}_{1} & \cdots & \boldsymbol{e}_{m-1}
\end{array}\right]^{\top} \\
\mathcal{C}_{*} & =\left[\begin{array}{llll}
\boldsymbol{e}_{1} & \mathbf{F}_{*} \boldsymbol{e}_{1} & \cdots & \mathbf{F}_{*}^{m-1} \boldsymbol{e}_{1}
\end{array}\right]
\end{aligned}
$$

where ${ }^{\top}$ denotes the matrix transpose operator. Let $\mathbf{F}=\mathbf{X F}_{*} \mathbf{X}^{-1}$ for an $m \times m$ nonsingular matrix $\mathbf{X}$, and let $\boldsymbol{g}$ be an $m \times 1$ vector such that $(\mathbf{F}, \boldsymbol{g})$ forms a controllable pair, i.e., the matrix

$$
\mathcal{C}:=\left[\begin{array}{llll}
g & \mathbf{F g} & \cdots & \mathbf{F}^{m-1} \boldsymbol{g}
\end{array}\right]
$$

is nonsingular. Then, for the polynomials $L\left(q^{-1}, \boldsymbol{\ell}\right)$ and $D\left(q^{-1}, \boldsymbol{\delta}\right)$ of the form given in (4) and (5)

$$
\begin{aligned}
\frac{L\left(q^{-1}, \boldsymbol{\ell}\right)}{D\left(q^{-1}, \boldsymbol{\delta}\right)} & =\boldsymbol{h}\left[\mathbf{I}_{m}-q^{-1} \mathbf{F}\right]^{-1} \boldsymbol{g} \\
& \Rightarrow \boldsymbol{h C}=\boldsymbol{\ell C}_{*} .
\end{aligned}
$$

Proof: See the Appendix.

Let $\mathbb{P}_{m}$ denote the vector space of order $m-1$ polynomials in $q^{-1}$ over the field $\mathbb{C}$ of complex numbers. We define the linear bijective map $\mathfrak{T}_{\mathbf{F}, \boldsymbol{g}}: \mathbb{C}^{1 \times m} \rightarrow \mathbb{P}_{m}$ as

$$
\mathfrak{T}_{\mathbf{F}, \boldsymbol{g}}(\boldsymbol{h})=h \mathcal{C C}_{*}^{-1} \boldsymbol{a}\left(q^{-1}\right), \quad \boldsymbol{h} \in \mathbb{C}^{1 \times m}
$$

where we define

$$
\boldsymbol{a}\left(q^{-1}\right):=\left[\begin{array}{llll}
1 & q^{-1} & \cdots & q^{-m+1}
\end{array}\right]^{\top} .
$$

Then, (14) in Proposition 1 can be written as $h\left[\mathbf{I}_{m}-\right.$ $\left.q^{-1} \mathbf{F}\right]^{-1} \boldsymbol{g}=\mathfrak{T}_{\mathbf{F}, \boldsymbol{g}}(\boldsymbol{h}) / D\left(q^{-1}, \boldsymbol{\delta}\right)$. Proposition 1 enables us to freeze the input-to-state filter in (7). First, we choose $\boldsymbol{g}$ and a suitable matrix $\mathbf{F}$ having $D\left(q^{-1}, \boldsymbol{\delta}\right)$ as its characteristic polynomial such that $\mathcal{C}$ in (13) is nonsingular. Then, it we must fine tune $\boldsymbol{h}$ in order to get an estimate of the annihilating numerator polynomial as [see (6) and (11)]

$$
L_{\star}\left(q^{-1}, \boldsymbol{\delta}\right)=\mathfrak{T}_{\mathbf{F}, \boldsymbol{g}}\left[\arg \min _{\boldsymbol{h}} \frac{\boldsymbol{h} \mathbf{Q} \boldsymbol{h}^{*}}{\boldsymbol{h E h ^ { * }}}\right] .
$$

Since we can keep $\mathbf{F}$ and $\boldsymbol{g}$ fixed, we can compute $\boldsymbol{x}(t)$ in (7), estimate $\mathbf{Q}$ as

$$
\widehat{\mathbf{Q}}=\frac{1}{N-p+1} \sum_{t=p}^{N} \boldsymbol{x}(t) \boldsymbol{x}^{*}(t)
$$

and replace $\mathbf{Q}$ in (17) by $\widehat{\mathbf{Q}}$. This is a good approximation for a moderately large data length $N$. Note that we have introduced a new integer $p$, which depends on the rate of decay of the transients [see Section II-D]. The idea is to allow sufficient time so that the transients due to IIR filtering decay to a sufficiently small value.

Next, we consider the minimization problem with respect to $\boldsymbol{h}$. If the filter order $m=d+1$, then the monic annihilating numerator polynomial is uniquely given by $A\left(q^{-1}, \boldsymbol{\omega}\right)$, but if we allow a larger order $m>d+1$, we have an $m-d$ dimensional subspace $\mathbb{S}_{m}(\boldsymbol{\omega})$ of $\mathbb{P}_{m}$ containing all the annihilating filter numerators, where

$$
\mathcal{B}_{m}(\boldsymbol{\omega})=\left\{q^{-k} A\left(q^{-1}, \boldsymbol{\omega}\right): k=0,1, \ldots, m-d-1\right\}
$$

forms a basis of $\mathbb{S}_{m}(\boldsymbol{\omega})$. Therefore, for a general $m$, it is preferable to compute an estimate of $\mathbb{S}_{m}(\boldsymbol{\omega})$ instead of a single annihilating numerator polynomial. Since $\mathfrak{T}_{\mathbf{F}, \boldsymbol{g}}$ is a bijective linear map, the problem of estimating $\mathbb{S}_{m}(\boldsymbol{\omega})$ is equivalent to the problem of estimating a subspace $\mathbb{Q}_{m}(\boldsymbol{\omega})$ of $\mathbb{C}^{1 \times m}$, where

$$
\mathbb{Q}_{m}(\boldsymbol{\omega}):=\left\{\boldsymbol{h} \in \mathbb{C}^{1 \times m}: \mathfrak{T}_{\mathbf{F}, \boldsymbol{g}}(\boldsymbol{h}) \in \mathbb{S}_{m}(\boldsymbol{\omega})\right\} .
$$

When we have a finite number of data samples $N$, we work with the estimated covariance matrix $\widehat{\mathbf{Q}}_{m}$. Then, it is reasonable to minimize the maximum SNR of $y_{f}(t)$ over all possible $m-$ $d$ dimensional subspaces $\mathbb{G}_{m-d}$ of $\mathbb{C}^{1 \times m}$. Consequently, we obtain an estimate $\hat{\mathbb{Q}}$ of $\mathbb{Q}_{m}(\boldsymbol{\omega})$ [see (11)] as

$$
\hat{\mathbb{Q}}:=\arg \min _{\mathbb{G}_{m-d}}\left[\max _{\boldsymbol{h} \in \mathbb{G}_{m-d}} \frac{\boldsymbol{h} \widehat{\mathbf{Q}} \boldsymbol{h}^{*}}{\boldsymbol{h} \mathbf{E} \boldsymbol{h}^{*}}\right] .
$$

The optimization problem (19) can be solved by solving the generalized eigenvalue problem [16]

$$
\widehat{\mathbf{Q}} \mathbf{u}=\lambda \mathbf{E u} .
$$

Let us denote the generalized eigenvectors of the matrix pencil $\widehat{\mathbf{Q}}-\lambda \mathbf{E}$ associated with the least $m-d$ eigenvalues by $\left\{\boldsymbol{u}_{k}\right\}_{k=1}^{m-d}$. Then, $\hat{\mathbb{Q}}=\operatorname{span}\left\{\boldsymbol{u}_{k}^{*}: k=1,2, \ldots, m-d\right\}$. Consequently, we obtain an estimate $\hat{\mathbb{S}}$ of $\mathbb{S}_{m}(\boldsymbol{\omega})$ (i.e., the subspace of annihilating numerator polynomials) as [see (15)]

$$
\begin{aligned}
\hat{\mathfrak{S}} & =\operatorname{span}\left\{\mathfrak{T}_{\mathbf{F}, \boldsymbol{g}}\left(\boldsymbol{u}_{k}^{*}\right): k=1,2, \ldots, m-d\right\} \\
& =\operatorname{span}\left\{\boldsymbol{u}_{k}^{*} \mathcal{C C}_{*}^{-1} \boldsymbol{a}\left(q^{-1}\right): k=1,2, \ldots, m-d\right\} .
\end{aligned}
$$

Recall that all the polynomials in $\mathbb{S}_{m}(\boldsymbol{\omega})$ have zeros at $\left\{e^{i \omega_{k}}\right\}_{k=1}^{d}$. Therefore, by inspecting the common minimum points of the frequency response functions of $\left\{\mathfrak{T}_{\mathbf{F}, \boldsymbol{g}}\left(\boldsymbol{u}_{k}^{*}\right)\right\}_{k=1}^{m-d}$, we can estimate the frequencies. However, due to small-sample estimation error in $\widehat{\mathbf{Q}}$, the zeros of the polynomials $\left\{\mathfrak{T}_{\mathbf{F}, \boldsymbol{g}}\left(\boldsymbol{u}_{k}^{*}\right)\right\}_{k=1}^{m-d}$ do not coincide in general. Therefore, it is required to perform some kind of averaging. This is done in the extended MUSIC algorithm in the following. Define the matrices

$$
\widehat{\mathbf{G}}=\left[\begin{array}{lll}
\boldsymbol{u}_{1} & \cdots & \boldsymbol{u}_{m-d}
\end{array}\right], \quad \widehat{\mathbf{S}}=\left[\begin{array}{lll}
\boldsymbol{u}_{m-d+1} & \cdots & \boldsymbol{u}_{m}
\end{array}\right]
$$

where $\left\{\boldsymbol{u}_{k}\right\}_{k=m-d+1}^{m}$ denotes the $d$ dominating generalized eigenvectors of the matrix pencil $\widehat{\mathbf{Q}}-\lambda \mathbf{E}$. We define the ex- 
tended MUSIC pseudo-spectrum as the sum of the reciprocals of the squared filter frequency responses:

$$
\begin{aligned}
& \sum_{k=1}^{m-d} \frac{\left|D\left(e^{-i \omega}, \boldsymbol{\delta}\right)\right|^{2}}{\left|\mathfrak{T}_{\mathbf{F}, \boldsymbol{g}}\left(\boldsymbol{u}_{k}^{*}\right)\right|^{2}} \\
& =\frac{\left|D\left(e^{-i \omega}, \boldsymbol{\delta}\right)\right|^{2}}{\boldsymbol{a}^{*}\left(e^{-i \omega}\right) \mathcal{C}_{*}^{-*} \mathcal{C}^{*} \widehat{\mathbf{G}} \widehat{\mathbf{G}}^{*} \mathcal{C C}_{*}^{-1} \boldsymbol{a}\left(e^{-i \omega}\right)} .
\end{aligned}
$$

We extend the Root-MUSIC algorithm in a similar fashion. We estimate the $d$ common zeros of the polynomials $\left\{\mathfrak{T}_{\mathbf{F}, \boldsymbol{g}}\left(\boldsymbol{u}_{k}^{*}\right)\right\}_{k=1}^{m-d}$ by computing the angular positions of the $d$ (pairs of reciprocal) roots of the equation

$$
\boldsymbol{a}^{\top}(q) \mathcal{C}_{*}^{-*} \mathcal{C}^{*} \widehat{\mathbf{G}} \widehat{\mathbf{G}}^{*} \mathcal{C C}_{*}^{-1} \boldsymbol{a}\left(q^{-1}\right)=0
$$

nearest to the unit circle. We show in Section II-B that the traditional MUSIC pseudo-spectrum [1], [8] is identical to (22) for a specific choice of $\mathbf{F}$ and $\boldsymbol{g}$. Similarly, the traditional RootMUSIC [9] is a special case of (23). Like the traditional MUSIC algorithm, we need to normalize the generalized eigenvectors. A natural way to normalize the generalized eigenvectors is as follows (which is quite common in many applications; see, for example, [17]):

$$
\left[\begin{array}{ll}
\widehat{\mathbf{S}} & \widehat{\mathbf{G}}
\end{array}\right]^{*} \mathbf{E}\left[\begin{array}{ll}
\widehat{\mathbf{S}} & \widehat{\mathbf{G}}
\end{array}\right]=\mathbf{I}_{m}, \quad\left[\begin{array}{ll}
\widehat{\mathbf{S}} & \widehat{\mathbf{G}}
\end{array}\right]^{*} \widehat{\mathbf{Q}}\left[\begin{array}{ll}
\widehat{\mathbf{S}} & \widehat{\mathbf{G}}
\end{array}\right]=\widehat{\mathbf{L}}
$$

where $\widehat{\mathbf{L}}$ is the diagonal matrix of the generalized eigenvalues arranged in proper order. We note in passing that the SNR minimization algorithm proposed here differs from the variant proposed in [12]. The most important consequence is the reduction in the number of eigenvalue problems to be solved. Here, we need to solve only one eigenvalue problem, whereas the algorithm in [12] needs to solve two eigenvalue problems. In Section II-C, we show that the proposed algorithm gives consistent frequency estimates.

We point out that the numerator in the expression for the extended spectral MUSIC in (22) is frequency dependent. In fact, one can choose to drop the numerator without disturbing the consistency of the frequency estimates. In that case, one has an alternative extended MUSIC pseudo-spectrum

$$
\frac{1}{\boldsymbol{a}^{*}\left(e^{-i \omega}\right) \mathcal{C}_{*}^{-*} \mathcal{C} * \widehat{\mathbf{G}} \widehat{\mathbf{G}}^{*} \mathcal{C C}_{*}^{-1} \boldsymbol{a}\left(e^{-i \omega}\right)} .
$$

However, it has been observed using numerical simulations that there is no significant difference between the performances of the versions (22) and (25).

\section{B. Further Insights on MUSIC}

The traditional MUSIC algorithm can be shown to be a special case of the extended MUSIC algorithm derived in Section II-A. FIR filters are employed in traditional MUSIC. It is shown in [12] that for the specific choice

$$
\mathbf{F}=\left[\begin{array}{llll}
\boldsymbol{e}_{2} & \cdots & \boldsymbol{e}_{m} & \boldsymbol{0}_{m \times 1}
\end{array}\right], \quad \boldsymbol{g}=\boldsymbol{e}_{1}
$$

the state vector $\boldsymbol{x}(t)=\left[\begin{array}{lll}y(t) & \cdots & y(t-m+1)\end{array}\right]^{\top}$, which is the standard snapshot vector. It is also readily verified from (10) that $\mathbf{E}=\mathbf{I}_{m}$ in this case. Therefore, the generalized eigenvalue problem in (20) reduces to a simple eigenvalue problem. Hence, the subspace $\hat{\mathbb{Q}}$ in (19) is spanned by the $m-d$ least eigenvectors of the covariance matrix $\widehat{\mathbf{Q}}$. Thus, when (26) holds, $\widehat{\mathbb{Q}}$ coincides with the the so-called estimated noise subspace [5]. It is readily verified by computing the characteristic polynomial of $\mathbf{F}$ in (26) that $D\left(q^{-1}, \boldsymbol{\delta}\right)=1$. Consequently, $\boldsymbol{\delta}=\mathbf{0}_{1 \times m}$. Therefore, a comparison between (12), (13), and (26) gives $\mathcal{C}_{*}=\mathcal{C}$ for this case. Using these observations in (22) gives the traditional MUSIC pseudo-spectrum [1], [5], [8]. Similarly, (23) reduces to the traditional Root-MUSIC equation [5], [9]. The traditional MUSIC algorithm uses the optimum annihilating FIR ${ }^{1}$ filters of order $m-1$, which minimize the SNR of $y_{f}(t)$. The MUSIC pseudo-spectrum is the sum of the reciprocals of the squared absolute frequency responses of the resulting filters. Therefore, the performance of the MUSIC algorithm is governed by the property of the underlying filters.

The SNR minimization approach to derive the MUSIC algorithm helps us to get meaningful insights. In Fig. 2(b), we have plotted the summed frequency response of the asymptotic MUSIC filters for different values of $m$. Here, $d=2$, and $\boldsymbol{\omega}=\left[\begin{array}{ll}1.4 & 1.5\end{array}\right]$. The values of $m$ in Fig. 1(a)-(c) are 3, 10, and 20, respectively. Clearly, the characteristics in Fig. 1(a)-(c) are associated with the corresponding annihilating FIR notch filters. Ideally, we want a notch characteristic as in Fig. 1(d). The noise energy blocked by the filter in Fig. 1(d) is minimal, which is required for robustness to additive noise. In addition, the selectivity of the filter in Fig. 1(d) is very high, which is required for a good resolution property. On the contrary, the characteristic in Fig. 1(a) (associated with the Pisarenko's harmonic decomposition method [15]) has a very wide valley. Notches are not even distinct. Such filters are not selective enough to provide a good resolution property. The wide valley causes the filter to block significant amounts of noise energy. This makes Pisarenko's method sensitive to additive noise. The situation improves as $m$ increases in Fig. 1(b) and (c). The width of the valley decreases, and the notches become more distinct. This is achieved by shaping the characteristics using the additional free zeros of the filter. Therefore, the performance improves as $m$ increases. In fact, we get reasonably distinct notches when we increase $m$ sufficiently. However, the valley width decreases at a very slow rate with $m$. Consequently, an extremely large value of $m$ is needed to achieve a sufficient degree of robustness to the additive noise. This is often impractical when the data length $N$ is finite. This explains why the good resolution property of MUSIC (and other subspace algorithms) with reasonably large $m$ is lost when data SNR is lowered.

A better quality of notch filters, as in Fig. 1(d), can be achieved by introducing poles close to the unit circle. It is well known that

$$
B\left(q^{-1}, r, \boldsymbol{\omega}\right):=\frac{A\left(q^{-1}, \boldsymbol{\omega}\right)}{A\left(r q^{-1}, \boldsymbol{\omega}\right)}, \quad 0 \leq r<1
$$

gives close to ideal notch characteristics as $r \rightarrow 1$. In Fig. 1(d), we used $r=0.99$. This is incorporated in the proposed algo-

\footnotetext{
${ }^{1}$ Since $D\left(q^{-1}, \delta\right)=1$ for the choice (26), all the poles are located at the origin.
} 
$\omega_{1}=1.4, \omega_{2}=1.5, \mathrm{~m}=3$

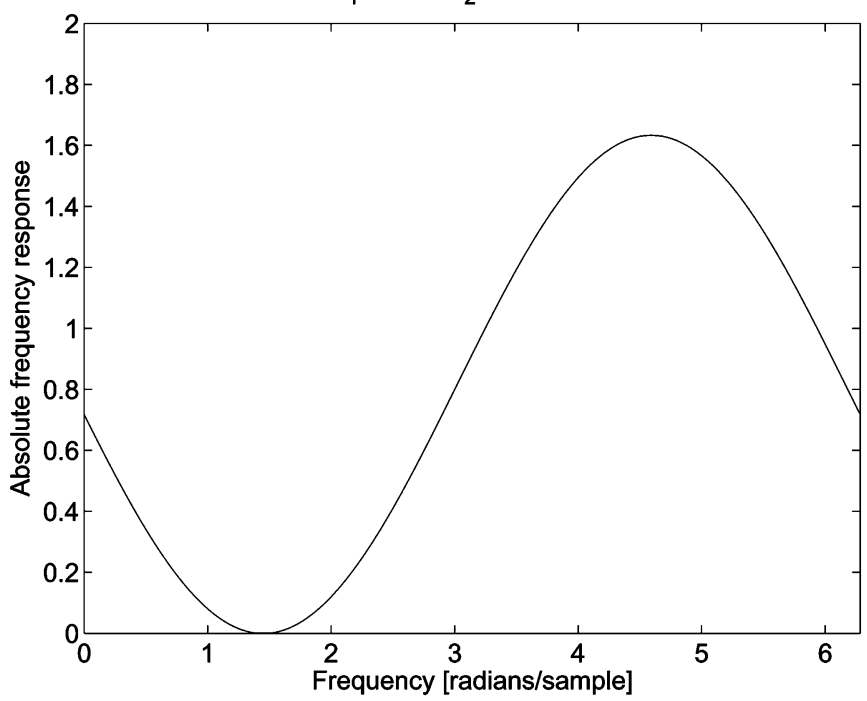

(a) Pisarenko's filter

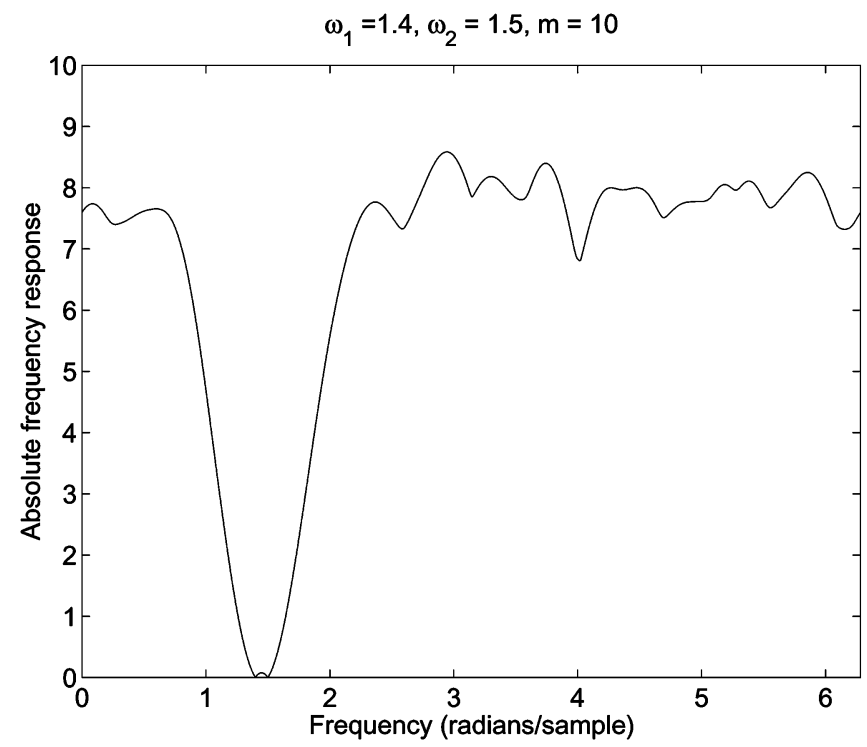

(b) Filter order 9

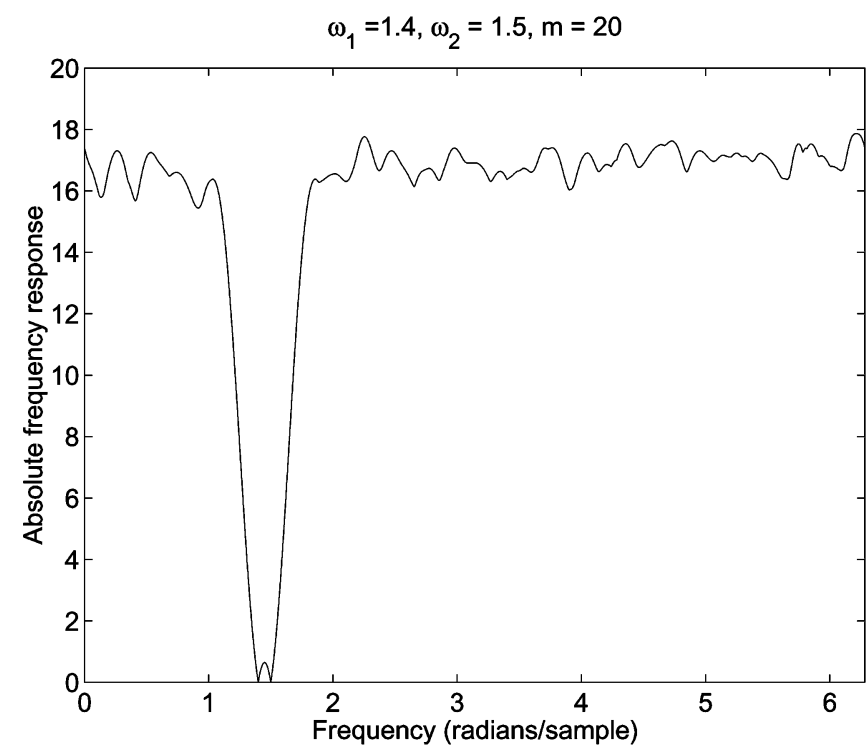

(c) Filter order 19

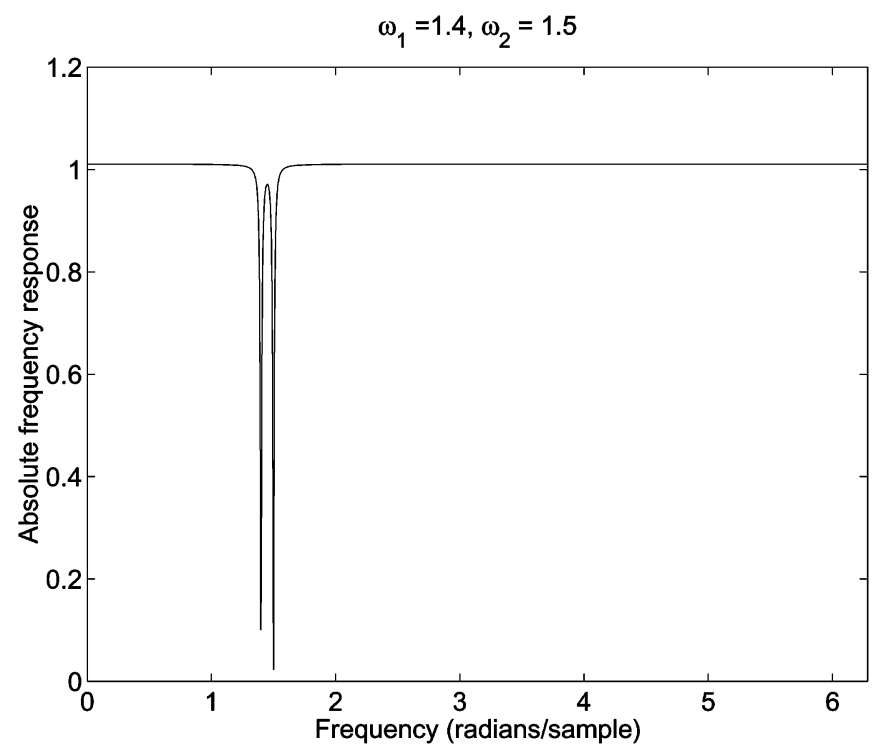

(d) Desired response

Fig. 1. Summed MUSIC filter frequency responses for $\omega=\left[\begin{array}{ll}1.4 & 1.5\end{array}\right]$. (a) $m=3$. (b) $m=10$. (c) $m=20$. A desired frequency response is shown in (d).

rithm by choosing a suitable $r$ and a corresponding $\mathbf{F}$. The idea of using such rational notch filters is not new. In fact, rational notch filters have been employed in the adaptive harmonic retrieval problem; see, for example, [18]-[21]. We also point out that a filter with a good frequency localization property has a long impulse response in time domain. Consequently, the parameter $p$ in (18) increases as $r \rightarrow 1$. More about this issue is highlighted in Section II-D.

In order to choose the good poles of the filters, we need to know $\boldsymbol{\omega}$, which might seem to be a problem with this approach. However, one key observation in this context plays the main role in simplifying this issue. That is, slight perturbations in the pole locations do not affect the nice properties of the filters. This motivates a two-step procedure. In the first step, one computes a crude ${ }^{2}$ estimate $\hat{\boldsymbol{\omega}}_{1}$ of $\boldsymbol{\omega}$ using a low-complexity estimator like the fast Fourier transform (FFT) or ESPRIT (more about this is given in Section II-D) and set [see (2) and (5)]

$$
\boldsymbol{\delta}=\left[\begin{array}{lll}
r a_{1}\left(\hat{\boldsymbol{\omega}}_{1}\right) & \cdots & r^{m} a_{m}\left(\hat{\boldsymbol{\omega}}_{1}\right)
\end{array}\right]
$$

Then, we realize the input-to-state filter (7) in controllable canonical form $\left(\mathbf{F}_{*}, \boldsymbol{e}_{1}\right)$; see (12) and (29). Note that we have extended the definition in (2):

$$
a_{k}(\boldsymbol{\omega})=0, \quad d<k \leq m .
$$

${ }^{2}$ In simulation examples in Section III, the estimation is carried out using very rough estimates of the pole locations. In fact, the length of $\hat{\omega}_{1}$ is less than $d$ in the implementation used to generate the simulation results in Section III. See Section II-D for details. 
The reason behind preferring the controllable canonical form will be clarified in Section II-D using some additional results derived in Section II-C.

\section{Analysis}

In this section, we derive certain invariance properties of the extended MUSIC algorithm. We also establish the strong consistency of the frequency estimates as the number of data samples $N \rightarrow \infty$. The notch filter interpretation in the previous section can be seen as a tool to choose the eigenvalues of $\mathbf{F}$. However, it is natural to ask whether a specific realization of $\mathbf{F}$ and $\boldsymbol{g}$ has any impact on the statistical properties of the estimates, which was kept open in [12] and [13]. We address this question in Proposition 4. We assume that the eigenvalues of $\mathbf{F}$ remain fixed in the subsequent analysis. We need the following results in Propositions 2 and 3 to prove Proposition 4.

Proposition 2: Let $\left(\mathbf{F}_{i}, \boldsymbol{g}_{i}\right)$ be controllable pairs for $i=1$ and 2 such that $\mathbf{F}_{2}=\mathbf{X F}_{1} \mathbf{X}^{-1}$ for a nonsingular matrix $\mathbf{X}$. Consider the input-to-state filters

$$
\boldsymbol{x}_{i}(t)=\mathbf{F}_{i} \boldsymbol{x}_{i}(t-1)+\boldsymbol{g}_{i} y(t) .
$$

Define the controllability matrices

$$
\mathcal{C}_{i}=\left[\begin{array}{llll}
\boldsymbol{g}_{i} & \mathbf{F}_{i} \boldsymbol{g}_{i} & \cdots & \mathbf{F}_{i}^{m-1} \boldsymbol{g}_{i}
\end{array}\right], \quad i=1,2 .
$$

Then, there exists a unique nonsingular matrix $\mathbf{T}=\mathcal{C}_{2} \mathcal{C}_{1}^{-1}$ such that both

$$
\boldsymbol{g}_{2}=\mathbf{T} \boldsymbol{g}_{1}, \quad \mathbf{T F}_{1}=\mathbf{F}_{2} \mathbf{T}
$$

are satisfied. As a consequence, $\boldsymbol{x}_{2}(t)=\mathbf{T} \boldsymbol{x}_{1}(t)$.

Proof: See [22, p. 107]. The proposition is a straightforward extension of the result proved in [22].

Proposition 3: Let $\lambda$ be a generalized eigenvalue of a matrix pencil $\mathbf{Q}-\rho \mathbf{E}$, and $\mathbf{u}$ be the eigenvector associated with $\lambda$. Suppose that $\mathbf{T}$ is any nonsingular matrix (of compatible dimension). Then, $\lambda$ is also a generalized eigenvalue of the matrix pencil TQT $^{*}-\rho \mathbf{T E T} \mathbf{T}^{*}$ with an associated eigenvector $\mathbf{v}=\mathbf{T}^{-*} \mathbf{u}$. Moreover

$$
\mathbf{u}^{*} \mathbf{E u}=1 \Rightarrow \mathbf{v}^{*}\left[\mathbf{T E T}^{*}\right] \mathbf{v}=1 \text {. }
$$

Proof: The proof is well known and, therefore, omitted.

Using the results in the previous propositions, we have the first result in this section in the form of the following proposition.

Proposition 4: If $(\mathbf{F}, \boldsymbol{g})$ is controllable, then the extended MUSIC estimate is invariant of the choice of $\mathbf{F}$ and $\boldsymbol{g}$, provided the characteristic polynomial of $\mathbf{F}$ remains the same.

Proof: Denote the state sequence obtained from the input-to-state filter in controllable canonical form by $\boldsymbol{x}_{*}(t)$. Therefore

$$
\boldsymbol{x}_{*}(t)=\mathbf{F}_{*} \boldsymbol{x}_{*}(t-1)+\boldsymbol{e}_{1} y(t)
$$

where $\mathbf{F}_{*}$ is defined in (12). Denote the noise contribution in $\boldsymbol{x}_{*}(t)$ by $\tilde{\boldsymbol{x}}_{*}(t)$. Introduce

$$
\begin{aligned}
& \widehat{\mathbf{Q}}_{*}=\frac{1}{N-p+1} \sum_{t=p}^{N} \boldsymbol{x}_{*}(t) \boldsymbol{x}_{*}^{*}(t) \\
& \mathbf{E}_{*}=\frac{1}{\sigma^{2}} \mathcal{E}\left\{\tilde{\boldsymbol{x}}_{*}(t) \tilde{\boldsymbol{x}}_{*}^{*}(t)\right\} .
\end{aligned}
$$

From Proposition 2, it follows that $\boldsymbol{x}_{*}(t)=\mathcal{C}_{*} \mathcal{C}^{-1} \boldsymbol{x}(t)$ [see (12) and (13)]. Therefore, we have

$$
\widehat{\mathbf{Q}}_{*}=\mathcal{C}_{*} \mathcal{C}^{-1} \widehat{\mathbf{Q}}^{-*} \mathcal{C}_{*}^{*}, \quad \mathbf{E}_{*}=\mathcal{C}_{*} \mathcal{C}^{-1} \mathbf{E} \mathcal{C}^{-*} \mathcal{C}_{*}^{*}
$$

Let $\widehat{\mathbf{G}}_{*}$ be the $m \times(m-d)$ matrix of $m-d$ least generalized eigenvectors of the matrix pencil $\widehat{\mathbf{Q}}_{*}-\rho \mathbf{E}_{*}$ as its columns. Then, from Proposition 3 and (30), we get

$$
\widehat{\mathbf{G}}=\mathcal{C}^{-*} \mathcal{C}_{*}^{*} \widehat{\mathbf{G}}_{*}
$$

Combining (31) with (22), we see that the extended MUSIC pseudo-spectrum satisfies

$$
\begin{aligned}
\frac{\left|D\left(e^{-i \omega}, \boldsymbol{\delta}\right)\right|^{2}}{\boldsymbol{a}^{*}\left(e^{-i \omega}\right) \mathcal{C}_{*}^{-*} \mathcal{C}^{*} \widehat{\mathbf{G}} \widehat{\mathbf{G}}^{*} \mathcal{C C}_{*}^{-1} \boldsymbol{a}\left(e^{-i \omega}\right)} \\
=\frac{\left|D\left(e^{-i \omega}, \boldsymbol{\delta}\right)\right|^{2}}{\boldsymbol{a}^{*}\left(e^{-i \omega}\right) \widehat{\mathbf{G}}_{*} \widehat{\mathbf{G}}_{*}^{*} \boldsymbol{a}\left(e^{-i \omega}\right)} .
\end{aligned}
$$

Similarly, the extended Root-MUSIC equation in (23) reduces to

$$
\boldsymbol{a}^{\top}(q) \widehat{\mathbf{G}}_{*} \widehat{\mathbf{G}}_{*}^{*} \boldsymbol{a}\left(q^{-1}\right)=0 .
$$

Now, (32) and (33) hold for any controllable $(\mathbf{F}, \boldsymbol{g})$, such that $\mathbf{F}$ and $\mathbf{F}_{*}$ have the same characteristic polynomial. Hence, the proposition follows.

In what follows next, we establish the consistency of the extended MUSIC estimate. The main idea is to extend the usual way of establishing the consistency of the conventional MUSIC algorithm. Toward that goal, we use (33). We point out that the consistency can also be established without using Proposition 4. Let us introduce

$$
\mathbf{Q}_{*} \mathcal{E}\left\{\boldsymbol{x}_{*}(t) \boldsymbol{x}_{*}^{*}(t)\right\}
$$

Clearly, as $N \rightarrow \infty$, the sample covariance matrix $\widehat{\mathbf{Q}}_{*}$ approaches $\mathbf{Q}_{*}$ with probability one. Consequently, the generalized eigenelements of the matrix pencil $\widehat{\mathbf{Q}}_{*}-\lambda \mathbf{E}_{*}$ approach the corresponding generalized eigenelements of the matrix pencil $\mathbf{Q}_{*}-\lambda \mathbf{E}_{*}$. We denote the limiting value of $\widehat{\mathbf{G}}_{*}$ by $\mathbf{G}_{*}$. Then, it is sufficient to show that

$$
\mathbf{G}_{*}^{*} \boldsymbol{a}\left(e^{-i \omega}\right)=0 \quad \text { iff } \omega=\omega_{k}, \quad k=1,2, \ldots, d .
$$

Let us introduce

$$
\mathbf{A}(\boldsymbol{\omega})=\left[\begin{array}{lll}
\boldsymbol{a}\left(e^{-i \omega_{1}}\right) & \cdots & \boldsymbol{a}\left(e^{-i \omega_{d}}\right)
\end{array}\right]
$$

Then, from standard results on the state-space models in controllable canonical form, one can verify, using (12) and (29), that

$$
\mathbf{Q}_{*}=\mathbf{A}(\boldsymbol{\omega}) \mathbf{P} \mathbf{A}^{*}(\boldsymbol{\omega})+\sigma^{2} \mathbf{E}_{*}
$$

where

$$
\mathbf{P}=\operatorname{diag}\left(\begin{array}{ccc}
\frac{c_{1}^{2}}{\mid D\left(e^{\left.-i \omega_{1}, \boldsymbol{\delta}\right)\left.\right|^{2}}\right.} & \cdots & \frac{c_{d}^{2}}{\mid D\left(e^{\left.-i \omega_{d}, \boldsymbol{\delta}\right)\left.\right|^{2}}\right.}
\end{array}\right)
$$


Let $\mathbf{S}_{*}$ be the $m \times d$ matrix having $d$ dominating generalized eigenvectors of the matrix pencil $\mathbf{Q}_{*}-\rho \mathbf{E}_{*}$ as its columns. Denote the $d \times d$ diagonal matrix of $d$ dominating generalized eigenvalues (arranged in proper order) by $\Lambda$. Similarly, the $(m-d) \times(m-d)$ diagonal matrix of $m-d$ least generalized eigenvalues is denoted by $\boldsymbol{\Sigma}$. Then, the normalization in (24) gives

$$
\begin{aligned}
& {\left[\begin{array}{l}
\mathbf{S}_{*}^{*} \\
\mathbf{G}_{*}^{*}
\end{array}\right] \mathbf{E}_{*}\left[\begin{array}{ll}
\mathbf{S}_{*} & \mathbf{G}_{*}
\end{array}\right]=\mathbf{I}_{m}} \\
& {\left[\begin{array}{l}
\mathbf{S}_{*}^{*} \\
\mathbf{G}_{*}^{*}
\end{array}\right] \mathbf{Q}_{*}\left[\begin{array}{ll}
\mathbf{S}_{*} & \mathbf{G}_{*}
\end{array}\right]=\left[\begin{array}{cc}
\Lambda & \mathbf{0}_{d \times(m-d)} \\
\mathbf{0}_{(m-d) \times d} & \Sigma
\end{array}\right] .}
\end{aligned}
$$

Hence, it is straightforward to derive that

$$
\mathbf{Q}_{*}=\mathbf{E}_{*} \mathbf{S}_{*} \Lambda \mathbf{S}_{*}^{*} \mathbf{E}_{*}+\mathbf{E}_{*} \mathbf{G}_{*} \boldsymbol{\Sigma} \mathbf{G}_{*}^{*} \mathbf{E}_{*} .
$$

Note that the eigenvalues of $\mathbf{E}_{*}^{-(1 / 2) *} \mathbf{Q}_{*} \mathbf{E}_{*}^{-(1 / 2)}$ are ${ }^{3}$ the same as the generalized eigenvalues of the matrix pencil $\mathbf{Q}_{*}-\lambda \mathbf{E}_{*}$. Therefore, we conclude from (36) that $\boldsymbol{\Sigma}=\sigma^{2} \mathbf{I}_{m-d}$. Now, using (36) and (37), we have

$$
\begin{aligned}
\mathbf{Q}_{*} \mathbf{S}_{*} & =\mathbf{E}_{*} \mathbf{S}_{*} \boldsymbol{\Lambda}=\mathbf{A}(\boldsymbol{\omega}) \mathbf{P} \mathbf{A}^{*}(\boldsymbol{\omega}) \mathbf{S}_{*}+\sigma^{2} \mathbf{E}_{*} \mathbf{S}_{*} \\
\Rightarrow \mathbf{E}_{*} \mathbf{S}_{*} & =\mathbf{A}(\boldsymbol{\omega}) \mathbf{P A}^{*}(\boldsymbol{\omega}) \mathbf{S}_{*}\left[\Lambda-\sigma^{2} \mathbf{I}_{d}\right]^{-1}
\end{aligned}
$$

Since $\mathbf{P} A^{*}(\boldsymbol{\omega}) \mathbf{S}_{*}\left[\boldsymbol{\Lambda}-\sigma^{2} \mathbf{I}_{d}\right]^{-1}$ is a $d \times d$ nonsingular matrix, we conclude that the column spaces of the matrices $\mathbf{E}_{*} \mathbf{S}_{*}$ and $\mathbf{A}(\boldsymbol{\omega})$ are identical. However, from (24), we see that the column spaces of $\mathbf{E}_{*} \mathbf{S}_{*}$ and $\mathbf{G}_{*}$ are mutually orthogonal to each other. Therefore, we conclude that

$$
\mathbf{G}_{*}^{*} \mathbf{A}_{*}(\boldsymbol{\omega})=\mathbf{0}_{(m-d) \times d} .
$$

Since both $\mathbf{G}_{*}$ and $\mathbf{A}(\boldsymbol{\omega})$ are full column rank matrices, combining (35) and (38), we get ${ }^{4}(34)$.

\section{Implementation Issues}

In this section, we highlight certain implementation aspects of the algorithm proposed in Section II-A. These are listed below.

Computation of $\hat{\boldsymbol{\omega}}_{1}$ : The primary frequency estimate $\hat{\boldsymbol{\omega}}_{1}$ need not be accurate. The basic idea is to find out the regions (on the frequency axis) where spectral lines are present. A fast Fourier transform (FFT) of the data can be used for this purpose, where we can just locate the peaks. Corresponding to each peak in the FFT, there exists a cluster of spectral lines to be resolved. Suppose that there exist $n$ clusters, and $d_{i}$ is the number of spectral peaks in the $i$ th cluster. This means that $d=\sum_{i=1}^{n} d_{i}$. If the number of spectral peaks $d_{i}$ in a candidate

\footnotetext{
${ }^{3}$ Since the pair $\left(\mathbf{F}_{*}, e_{1}\right)$ is controllable, $\mathbf{E}_{*}$ is positive definite. Therefore, the Cholesky factor $\mathbf{E}_{*}^{(1 / 2)}$ exists, and it is nonsingular.

${ }^{4}$ Strictly speaking, one also needs establish the parameter identifiability, i.e., (38) cannot be satisfied by another set of frequencies. However, conditions for this to hold can be found in the literature; see, for example, [23].
}

cluster $i$ is a priori known (for example, in certain nuclear magnetic resonance spectroscopy applications [24]), then we can place $d_{i}$ multiple entries of the center frequency of cluster $i$ in the $\hat{\omega}_{1}$ vector. Otherwise, we can set $d_{i}=1$ and estimate $d$ from the generalized eigenspectrum of the matrix pencil $\widehat{\mathbf{Q}}-\rho \mathbf{E}$ using methods similar to [25] and [26]. However, a detailed treatment on the detection problem is out of the scope of this paper. If the data SNR is very low, an FFT estimate of the cluster center frequencies might be erroneous. In that case, one can use an ESPRIT algorithm to estimate the center frequencies, pretending that only $n$ sinusoids are present in the data. In the simulation examples presented in Section III, we have $n=2, d_{1}=3$, and $d_{2}=2$. We have used the ESPRIT algorithm to determine the cluster center frequencies. Subsequently, we construct $\hat{\boldsymbol{\omega}}_{1}$, assuming $d_{1}=1$ and $d_{2}=1$. Thus, $\hat{\boldsymbol{\omega}}_{1}$ becomes a $2 \times 1$ vector composed of the center frequency estimates, which is then used to compute $\delta$, as in (27).

Choice of $r$ and $p$ : The choice of $r$ is governed by the number of available data points $N$, as well as the data SNR. The larger the value of $N$, the larger the value of $r$ will be. On the other hand, larger the data SNR, the smaller the value of $r$ will be. The reason is straightforward. A value of $r$ close to unity results longer transients. Therefore, using $r$ close to unity for small $N$ leads to loss of data points due to transients. On the other hand, with increased noise level, it is desired to have smaller notch width of the notch filter. This requires $r$ more close to unity. In Section III, we illustrate this phenomenon using numerical simulation results. The choice of the parameter $p$ is directly related to the rate of decay of the transients. In fact, it is easy to show that $p$ should be related logarithmically to $r$. In the examples in Section III, we have used

$$
p=\frac{-0.5}{\ln r} .
$$

Forward and Backward Filtering: Let $\bar{y}_{0}(t)$ be the complex conjugate of $y_{0}(t)$. Define

$$
y_{0}(t)=\bar{y}_{0}(N-t+1) \text {. }
$$

Then, it is straightforward to verify that $y_{0}(t)$ and $y_{0}(t)$ have the identical spectral characteristics. This fact can be exploited to double the number of the snapshot vectors available for the covariance computation (in principle, this is similar to the concept introduced in [27]). We also pass the sequence $y(t)$ [the noise-corrupted version of $\left.y_{0}(t)\right]$ through the input-to-state filter (7) to obtain $\boldsymbol{x}(t)$ :

$$
\dot{\boldsymbol{x}}(t)=\mathbf{F} \boldsymbol{x}(t-1)+\boldsymbol{g} \dot{y}(t) .
$$

Now, $\{\dot{x}(t)\}_{t=p}^{N}$ can also be used for covariance computation.

Choice of Coordinates and Forward-Backward Averaging: In the previous section, we established that any specific choice of $\mathbf{F}$ and $\boldsymbol{g}$ has no impact on the extended MUSIC estimates. However, $\mathbf{F}$ and $\boldsymbol{g}$ can be chosen to impose a 
TABLE I

OUTLINE OF THE EXTENDED MUSIC ALGORITHM

1. Compute the cluster centre frequencies using an FFT or an ESPRIT algorithm.

2. Form the vector $\hat{\boldsymbol{\omega}}_{1}$. Choose $r$ and compute $p$ using (39).

3. Compute $\delta$ using (27), $\mathbf{F}_{*}$ using (12), and $\mathbf{E}_{*}$ by solving

$$
\mathbf{E}_{*}=\mathbf{F}_{*} \mathbf{E}_{*} \mathbf{F}_{*}+e_{1} \boldsymbol{e}_{1}^{*} .
$$

4. Compute the state vectors $\boldsymbol{x}_{*}(t)$ and $\dot{\boldsymbol{x}}_{*}(t)$ such that

$$
\begin{aligned}
& \boldsymbol{x}_{*}(t)=\mathbf{F}_{*} \boldsymbol{x}(t-1)+\boldsymbol{e}_{1} y(t) ; \\
& \dot{x}_{*}(t)=\mathbf{F}_{*} \dot{\boldsymbol{x}}_{*}(t-1)+\boldsymbol{e}_{1} \dot{y}(t) .
\end{aligned}
$$

5. Compute $\widehat{\mathbf{Q}}_{*}=\frac{1}{2(N-p+1)} \sum_{t=p}^{N}\left\{\boldsymbol{x}_{*}(t) \boldsymbol{x}_{*}^{*}(t)+\dot{\boldsymbol{x}}_{*}(t) \dot{\boldsymbol{x}}_{*}^{*}(t)\right\}$, and form $\widehat{\mathbf{Q}}_{*}^{a}=\frac{1}{2}\left(\widehat{\mathbf{Q}}_{*}+\mathbf{J}_{m} \widehat{\mathbf{Q}}_{*}^{\top} \mathbf{J}_{m}\right)$.

6. Compute $\widehat{\mathbf{G}}_{*}$ from the least $m-d$ eigenvectors of the matrix pencil $\widehat{\mathbf{Q}}_{*}^{a}-\lambda \mathbf{E}_{*}$.

7. Compute the extended MUSIC pseudo-spectrum using the right hand side of (32) and (16).

8. Extended Root-MUSIC estimates are computed by rooting the equation (33).

centro-symmetric structure 5 on $\mathbf{Q}$. The centro-symmetric covariance structure can then be exploited to obtain a forward-backward averaged covariance matrix [3], [28]

$$
\widehat{\mathbf{Q}}^{a}=\frac{1}{2}\left(\widehat{\mathbf{Q}}+\mathbf{J}_{m} \widehat{\mathbf{Q}}^{\top} \mathbf{J}_{m}\right)
$$

where $\mathbf{J}_{m}$ denotes the $m \times m$ permutation matrix having ones along its anti-diagonal and zeros elsewhere. In small samples, the forward-backward averaging is known to give a significant improvement in the estimation accuracy [3], [28]. One way to impose the centro-symmetric structure on $\mathbf{Q}$ is to use the controllable canonical form in (29). It is well known for a controllable canonical form that the state vector $\boldsymbol{x}_{*}(t)$ is composed of the delayed versions of the filtered signal $\left\{1 / D\left(q^{-1}, \boldsymbol{\delta}\right)\right\} y(t)$. Consequently, $\mathbf{Q}_{*}$ is a Hermitian Toeplitz matrix, which is centro-symmetric.

Computation of $\mathbf{E}_{*}$ : We can compute $\mathbf{E}_{*}$ by solving a Lyapunov equation in (10). However, if the controllable canonical form is used, it is possible to compute $\mathbf{E}_{*}$ using an FFT algorithm. The idea is to compute the autocorrelation sequence of $\left\{1 / D\left(q^{-1}, \boldsymbol{\delta}\right)\right\} \tilde{y}(t)$ as the inverse Fourier transform of the spectral density. There are other simple methods based on the half spectrum of $\left\{1 / D\left(q^{-1}, \delta\right)\right\} \tilde{y}(t)$. See [29] and references therein for details.

An outline of the extended MUSIC algorithm is given in Table I.

\section{NUMERICAL ILLUSTRATION}

In this section, we illustrate the performance of the proposed algorithm using numerical simulation results. In the examples, we have $\boldsymbol{\omega}=\left[\begin{array}{lllll}1.3 & 1.4 & 1.5 & 2.4 & 2.5\end{array}\right]$, and the number of

\footnotetext{
${ }^{5} \mathrm{An} m \times m$ matrix $\mathbf{X}$ is centro-symmetric if $\mathbf{X}=\mathbf{J}_{m} \check{\mathbf{X}} \mathbf{J}_{m}$, where $\check{\mathbf{X}}$ is the complex conjugate of $\mathbf{X}$.
}

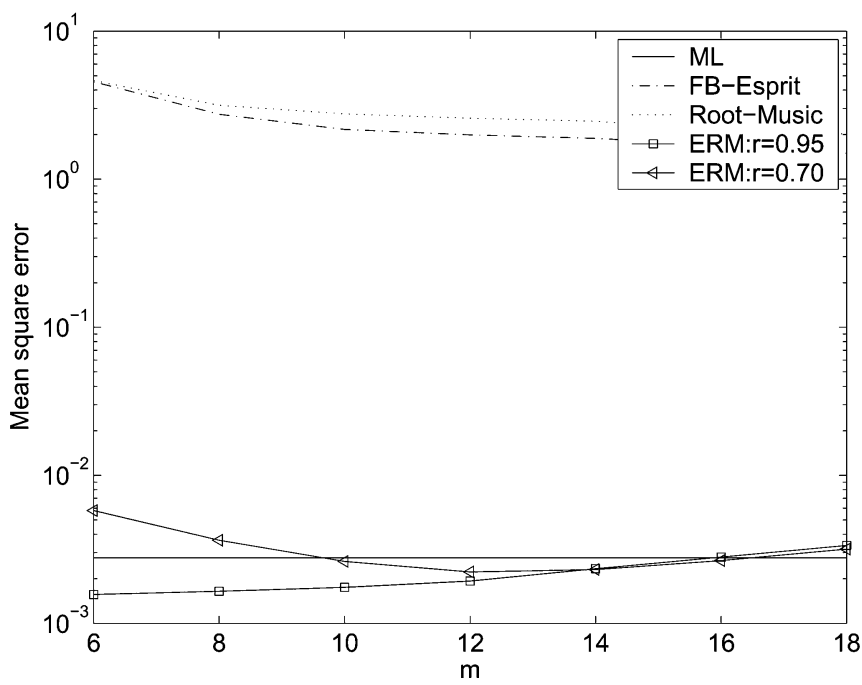

(a) Average MSE for cluster-1

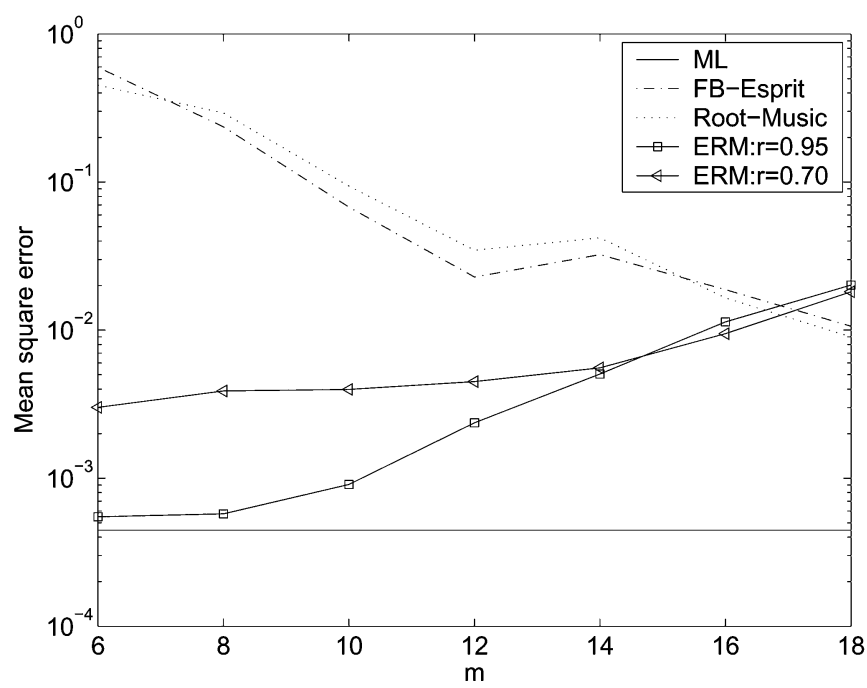

(b) Average MSE for cluster-2

Fig. 2. Average MSE for different algorithms as functions of $m$ for two frequency clusters at $10 \mathrm{~dB}$ SNR. (a) First frequency cluster corresponding to $\omega_{1}=1.3, \omega_{2}=1.4$, and $\omega_{3}=1.5$. (b) Second frequency cluster corresponding to $\omega_{4}=2.4$ and $\omega_{5}=2.5$.

data samples $N=35$. Note that the corresponding Fourier resolution is $(2 \pi / 35)=0.18$. This is 1.8 times the minimum spacing between the frequencies under consideration. The amplitudes $\left\{c_{k}\right\}_{k=1}^{5}$ are set to unity. The performance of the extended Root-MUSIC (ERM) algorithm is compared with the maximum likelihood (ML) method [5], traditional Root-MUSIC, and the forward-backward ESPRIT algorithm. Note that the ML method involves a nonlinear least squares optimization.

Here, we have two clusters of frequencies. In the first cluster, we have three frequencies: $\omega_{1}=1.3, \omega_{2}=1.4$, and $\omega_{3}=1.5$. The other cluster contains two remaining frequencies $\omega_{4}=2.4$ and $\omega_{5}=2.5$. In the implementation of the ERM algorithm, the cluster center frequencies are estimated using an ESPRIT algorithm, where we pretend that only two frequencies are present in the data. Denote these two preliminary cluster center frequency estimates by $\varpi_{1}$ and $\varpi_{2}$. Subsequently, we assume that every 


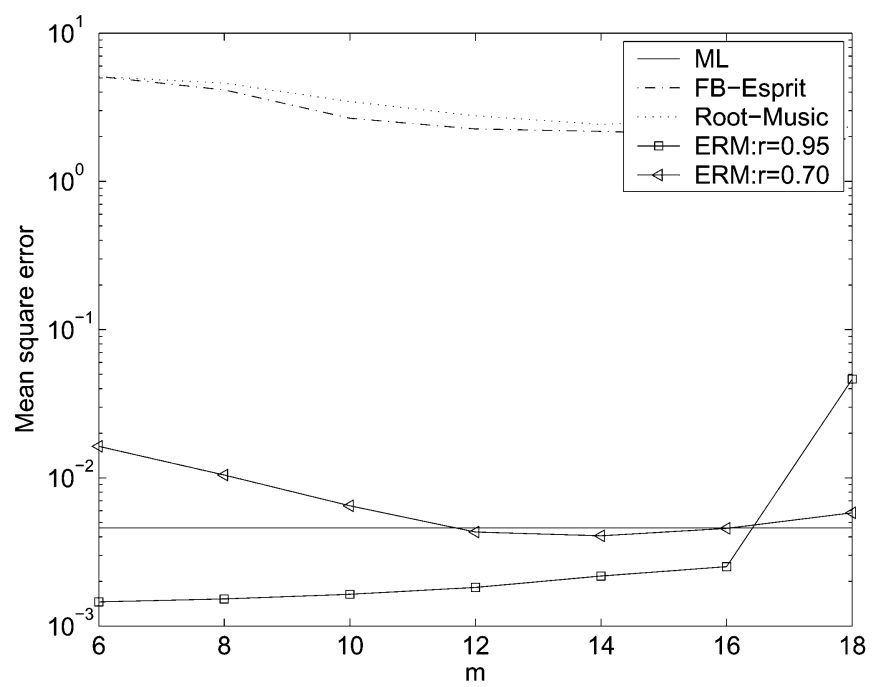

(a) Average MSE for cluster-1

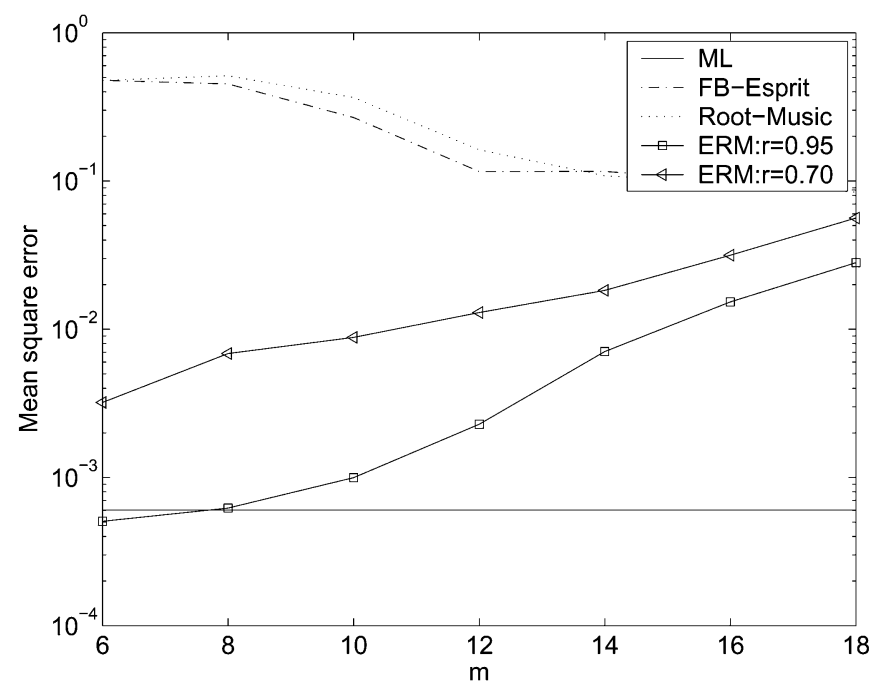

(b) Average MSE for cluster-2

Fig. 3. Average MSE for different algorithms as functions of $m$ for two frequency clusters at $5 \mathrm{~dB}$ SNR. (a) First frequency cluster corresponding to $\omega_{1}=1.3, \omega_{2}=1.4$ and $\omega_{3}=1.5$. (b) Second frequency cluster corresponding to $\omega_{4}=2.4$ and $\omega_{5}=2.5$.

cluster contains only one frequency point, i.e., $d_{1}=1$ and $d_{2}=1$. Therefore, we set

$$
\hat{\boldsymbol{\omega}}_{1}=\left[\begin{array}{lllll}
\varpi_{1} & \varpi_{2} & 0 & \cdots & 0
\end{array}\right]
$$

and compute $\delta$ and $\mathbf{F}_{*}$ using (27) and (12), respectively.

In Fig. 2, we show the estimation results for a 10-dB SNR $\left(\sigma^{2}=0.1\right)$. We plot mean square estimation errors (MSE) using different algorithms as functions of snapshot size $m$. The results for ERM algorithm is shown for $r=0.95$ and $r=0.70$. In Fig. 2(a), we have plotted the average MSE of the frequency estimates in the first cluster ${ }^{6}$, i.e., $\omega_{1}=1.3, \omega_{2}=1.4$, and $\omega_{3}=1.5$. Similarly, the average MSE associated with the frequencies $\omega_{4}=2.4$ and $\omega_{5}=2.5$ in the second cluster are depicted in Fig. 2(b). The numerical MSE are based on estima-

${ }^{6}$ The results for each individual frequency in a particular cluster are similar. An average MSE carries approximately the same information.

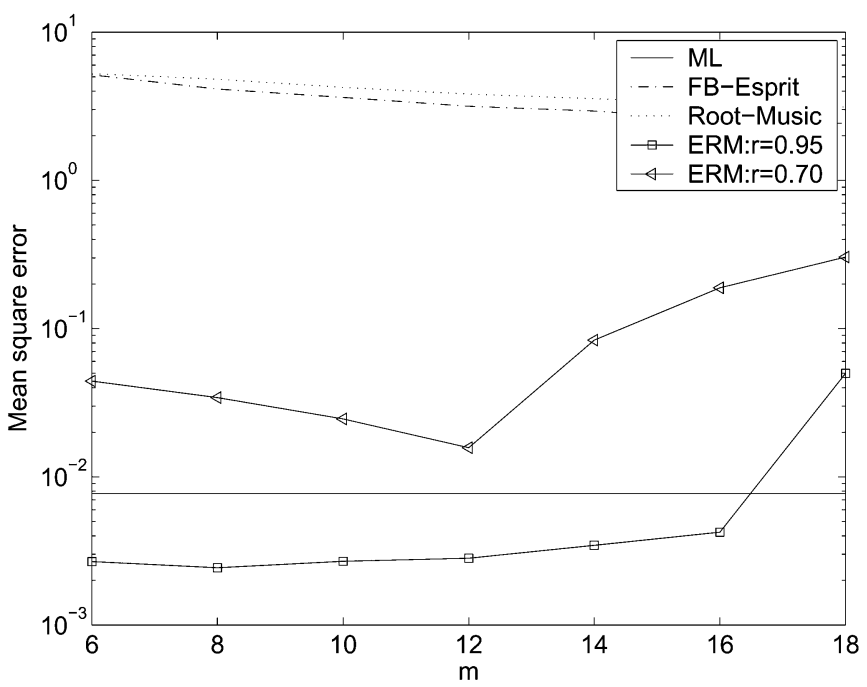

(a) Average MSE for cluster-1

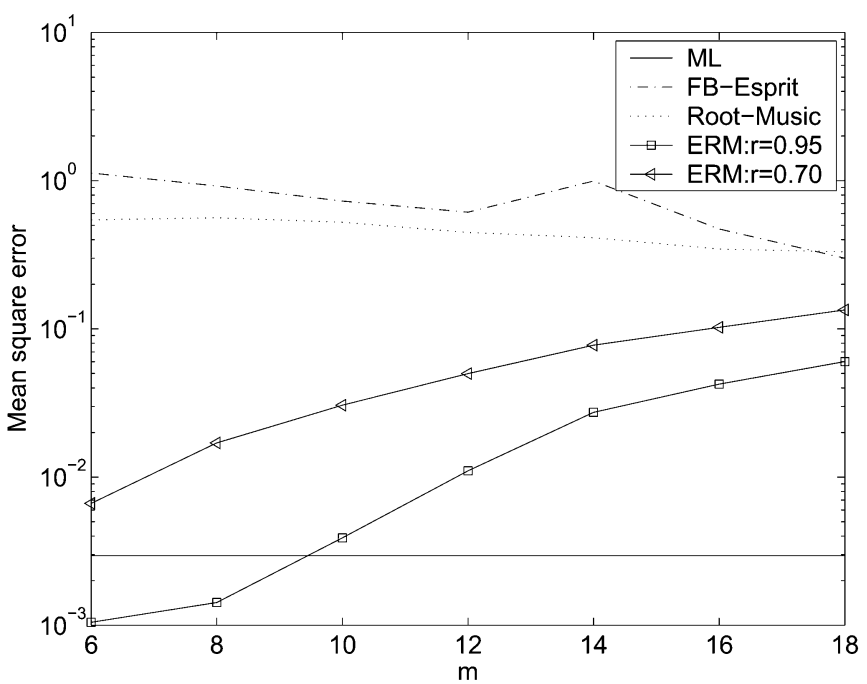

(b) Average MSE for cluster-2

Fig. 4. Average MSE for different algorithms as functions of $m$ for two frequency clusters at $0 \mathrm{~dB}$ SNR. (a) First frequency cluster corresponding to $\omega_{1}=1.3, \omega_{2}=1.4$, and $\omega_{3}=1.5$. (b) Second frequency cluster corresponding to $\omega_{4}=2.4$ and $\omega_{5}=2.5$.

tion results in 100 independent Monte Carlo simulations. In the simulations, we initialize the numerical optimization for computing the ML estimates at the true parameter values. ${ }^{7}$ The estimation results obtained from 100 Monte Carlo simulations at $5 \mathrm{~dB}$ SNR $\left(\sigma^{2}=0.31\right)$ are displayed in Fig. 3, where the estimations results for the first and the second frequency clusters are shown in Fig. 3(a) and (b), respectively. A similar performance comparison for $0 \mathrm{~dB}$ SNR $\left(\sigma^{2}=1\right)$ is given in Fig. 4 . Recall that the minimum frequency spacing in either of the two frequency clusters is 0.1 . Therefore, an average MSE more than 0.01 in a frequency cluster indicates that the corresponding algorithm is unable to resolve the frequencies in that cluster. In

\footnotetext{
${ }^{7}$ It is generally required to initialize the optimization to compute the ML estimate close to the true parameters. In a practical scenario, the initial point is found by an algorithm like ESPRIT, which does not involve any search [6]. When the data SNR is low, however, the initial point obtained in this fashion may be erroneous; see the results in Figs. 2-4. For this reason, we initialize the optimization at the true parameter values. However, we caution that the performance of the ML algorithm shown in Figs. 2-4 may not be achievable in a practical scenario.
} 

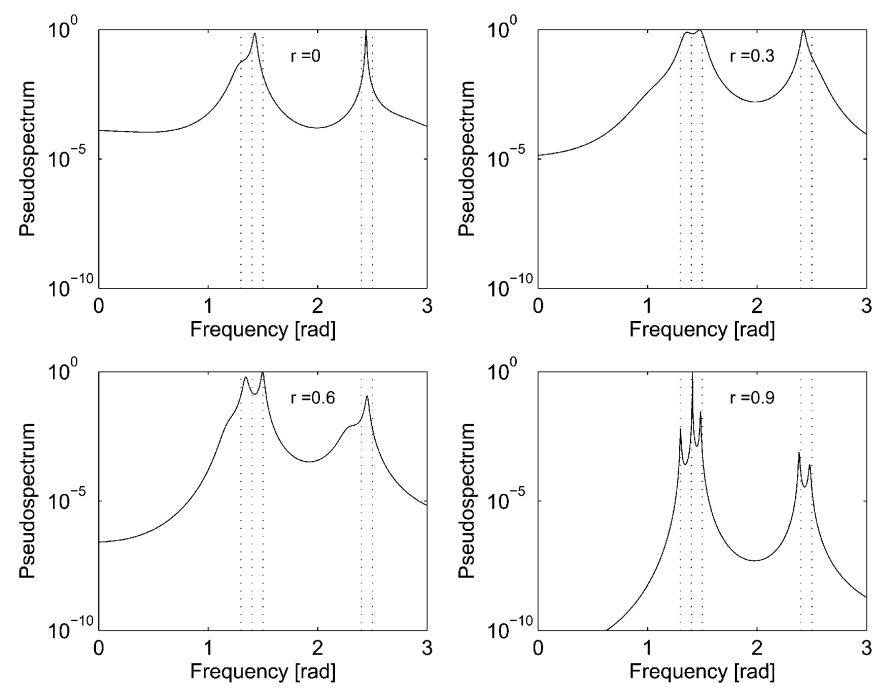

Fig. 5. Evolution of the extended MUSIC pseudo-spectrum with increase in $r$ for $m$ fixed at 12. The plot for $r=0$ at the top left, $r=0.3$ at the top right, $r=0.6$ at the bottom left, and $r=0.9$ at the bottom right. The estimated pseudo-spectrum in solid lines is compared with the true frequency locations in the dotted line.

that view, we note in Figs. 2-4 that neither Root-Music nor forward-backward ESPRIT is capable of resolving the frequencies when data $\mathrm{SNR} \leq 10 \mathrm{~dB}$. For the frequency cluster- 1 composed of $\omega_{1}=1.3, \omega_{2}=1.4$ and $\omega_{3}=1.5$, the ERM algorithm for $r=0.95$ outperforms the ML algorithm. In the frequency cluster- 2 composed of $\omega_{4}=2.4$ and $\omega_{5}=2.5$, ML is slightly better than Extended Root-MUSIC (ERM) $(r=0.95)$ at $10 \mathrm{~dB}$ SNR. However, for smaller SNR values, ERM corresponding to $r=0.95$ also outperforms ML in the second frequency cluster. ${ }^{8}$ Note that the estimation problem is more difficult in cluster-1 because it has three closely spaced frequencies compared to two frequencies in cluster-2. It is also interesting to note in Figs. 2(a), 3(a), and 4(a) that the performance of the extended MUSIC algorithm for larger $r$ is better for smaller values of snapshot dimension $m$. On the other hand, maintaining a smaller $r$ needs larger $m$ to achieve the best performance. This is in agreement with our discussion in Sections II-B and D. Maintaining $r$ close to unity, we have sharp notch filters for small values of $m$ at the expense of long transients. Due to long transients, the noise-free part of the filter output $y_{f}(t)$ behaves like sinusoids with slowly varying amplitude [30]-[32]. Hence, for a larger $m$, the snapshot vectors $\boldsymbol{x}_{*}(t)$ do not lie in the column space of $\mathbf{A}(\boldsymbol{\omega})$. The problem is less pronounced if the number of data samples is large compared with the time constant of the filter $1 / A\left(r q^{-1}, \hat{\boldsymbol{\omega}}_{1}\right)$.

In Fig. 5, we have shown the extended MUSIC pseudo-spectrum plots obtained in one realization. Here, the estimated pseudo-spectrum are compared with the true frequency locations (plotted in dotted lines). The SNR in this realization is $0 \mathrm{~dB}$, and the number of data samples is 35 . Here, we consider the extended MUSIC pseudo-spectrum for $r=0, r=0.3$, $r=0.6$, and $r=0.9$ in the frequency range $0-3 \mathrm{rad}$. Recall that the plot corresponding to $r=0$ gives the traditional MUSIC pseudo-spectrum. In Fig. 5, it is interesting note how

\footnotetext{
${ }^{8}$ This indicates that the ERM algorithm is more robust to outliers in comparison to ML. Note that at lower SNR the probability of getting an outlier is high.
}

the pseudo-spectrum evolves with the increase in $r$. It is also interesting to note that all the frequencies are resolved for $r=0.9$. We also point out that for $r=0$ and $r=0.3$, the two different clusters can be recognized clearly, but the individual frequencies within a cluster cannot be resolved.

\section{CONCLUSIONS}

In this paper, we have presented a novel interpretation of the MUSIC algorithm for the spectrum estimation problem. It is shown that the classical MUSIC algorithm is a data-dependent notch filter design method. However, the MUSIC filters in the classical framework are FIR. Therefore, they do not have adequate localization property in the frequency domain, unless the filter length $m$ is very large. Working with a finite number of data samples, it is often impractical because that leads to a significant reduction in the number of snapshot vectors used for covariance computation. Hence, traditional MUSIC has limitations in resolution property. The performance is poor in particular if the SNR is low. To remedy this problem, we have introduced the rational notch filters and extended MUSIC in this framework. The rational notch filters have excellent localization property in frequency domain. In the sequel, an improved algorithm using generalized input-to-state filters is proposed. The proposed algorithm needs to solve only one eigenvalue problem instead of two eigenvalue problems in [12] and [13] and, hence, is more efficient computationally. We have also established the strong consistency of the proposed algorithm. It turns out that the extended MUSIC estimate is independent of the choice of coordinates. The proposed algorithms exhibit significant improvement in the resolution performance. It is robust to the additive noise as well. At low SNR, the proposed algorithm outperforms the maximum likelihood method.

\section{APPENDIX}

PROOF OF PROPOSITION 1

In this Appendix, we write $\mathbf{T}=\mathcal{C C}_{*}^{-1}$. The proof will use Proposition 2. Note that from Proposition 2, it follows that $\mathbf{T}$ is the unique nonsingular matrix such that

$$
g=\mathbf{T} e_{1}, \quad \mathbf{F T}=\mathbf{T F}_{*} \Rightarrow \mathbf{F}_{*} \mathbf{T}^{-1}=\mathbf{T}^{-1} \mathbf{F} .
$$

Hence, we see that

$$
\begin{aligned}
& \frac{L\left(q^{-1}, \boldsymbol{\ell}\right)}{D\left(q^{-1}, \boldsymbol{\delta}\right)}=\boldsymbol{h}\left[\mathbf{I}_{m}-q^{-1} \mathbf{F}\right]^{-1} \boldsymbol{g} \\
\Rightarrow & \frac{L\left(q^{-1}, \boldsymbol{\ell}\right)}{D\left(q^{-1}, \boldsymbol{\delta}\right)}=\boldsymbol{h}\left[\mathbf{T}^{-1}-q^{-1} \mathbf{T}^{-1} \mathbf{F}\right]^{-1} \boldsymbol{e}_{1} \\
\Rightarrow & \frac{L\left(q^{-1}, \boldsymbol{\ell}\right)}{D\left(q^{-1}, \boldsymbol{\delta}\right)}=\boldsymbol{h}\left[\mathbf{T}^{-1}-q^{-1} \mathbf{F}_{*} \mathbf{T}^{-1}\right]^{-1} \boldsymbol{e}_{1} \\
\Rightarrow & \frac{L\left(q^{-1}, \boldsymbol{\ell}\right)}{D\left(q^{-1}, \boldsymbol{\delta}\right)}=\boldsymbol{h} \mathbf{T}\left[\mathbf{I}_{m}-q^{-1} \mathbf{F}_{*}\right]^{-1} \boldsymbol{e}_{1} \\
\Rightarrow & \frac{L\left(q^{-1}, \boldsymbol{\ell}\right)}{D\left(q^{-1}, \boldsymbol{\delta}\right)}=\frac{\boldsymbol{h} \mathbf{T} \boldsymbol{a}\left(q^{-1}\right)}{D\left(q^{-1}, \boldsymbol{\ell}, \boldsymbol{\delta}\right)}
\end{aligned}
$$

where in the last equality, we have used the property of a controllable canonical form. Therefore, equating the numerators in both sides of the last equality and using (4), (5), and (16), we get

$$
\boldsymbol{\ell a}\left(q^{-1}\right)=\boldsymbol{h} \mathbf{T a}\left(q^{-1}\right) \text {. }
$$


Thus, the proposition follows by equating the coefficients of $\left\{q^{-k}\right\}_{k=0}^{m-1}$.

\section{ACKNOWLEDGMENT}

The author would like to thank the anonymous reviewers for many valuable comments and suggestions, which helped to improve the quality of the paper significantly.

\section{REFERENCES}

[1] R. O. Schimidt, "Multiple emitter location and signal parameter estimation," IEEE Trans. Antennas Propag., vol. AP-34, no. 3, pp. 276-280, Mar. 1986.

[2] R. H. Roy, "ESPRIT-a subspace rotation approach to estimation of parameters of cisoids in noise," IEEE Trans. Acoust., Speech Signal Process., vol. ASSP-34, no. 10, pp. 1340-1342, Oct. 1986.

[3] A. Eriksson, P. Stoica, and T. Söderström, "Markov-based eigenanalysis method for frequency estimation," IEEE Trans. Signal Process., vol. 42, no. 3, pp. 586-594, Mar. 1994.

[4] M. Kristensson, M. Jansson, and B. Ottersten, "Further results and insights on subspace based sinusoidal frequency estimation," IEEE Trans. Signal Process., vol. 49, no. 12, pp. 2962-2974, Dec. 2001.

[5] P. Stoica and R. Moses, Introduction to Spectral Analysis, Second ed. Upper Saddle River, NJ: Prentice-Hall, 2004.

[6] P. Stoica, R. Moses, B. Friedlander, and T. Söderström, "Maximum likelihood estimation of the parameters of multiple sinusoids from noisy measurements," IEEE Trans. Acoust., Speech, Signal Process., vol. 37, no. 3, pp. 378-392, Mar. 1989.

[7] P. Stoica and T. Söderström, "Statistical analysis of MUSIC and subspace rotation estimates of sinusoidal frequencies," IEEE Trans. Signal Process., vol. 39, no. 8, pp. 1836-1847, Aug. 1991.

[8] G. Bienvenu, "Influence of the spatial coherence of the background noise on high resolution passive methods," in Proc. ICASSP, Washington, DC, 1979, pp. 306-309.

[9] A. J. Barabell, "Improving the resolution performance of eigenstructure-based direction-finding algorithms," in Proc. ICASSP, Boston, MA, 1983, pp. 336-339.

[10] B. D. Rao and K. V. S. Hari, "Perfomance analysis of root-MUSIC," IEEE Trans. Signal Process., vol. 37, no. 2, pp. 1939-1949, Feb. 1989.

[11] C. I. Byrnes, T. T. Georgiou, and A. Lindquist, "A new approach to spectral estimation: A tunable high-resolution spectral estimator," IEEE Trans. Signal Process., vol. 48, no. 11, pp. 3189-3205, Nov. 2000.

[12] T. T. Georgiou, "Signal estimation via selective harmonic amplification: MUSIC, redux," IEEE Trans. Signal Process., vol. 48, no. 3, pp. 780-790, Mar. 2000

[13] — "Spectral estimation via selective harmonic amplification," IEEE Trans. Autom. Control, vol. 46, no. 1, pp. 29-42, Jan. 2001.

[14] L. Tong, G. Xu, and T. Kailath, "A new approach to blind identification and equalization of multipath channels," in Proc. 25th Asilomar Conf. Signals, Syst. Comput., Pacific Grove, CA, Nov. 1991, pp. 856-860.

[15] V. F. Pisarenko, "The retrieval of harmonics from a covariance function," Geophys. J. R. Astron. Soc., vol. 33, pp. 347-366, 1973.

[16] G. Strang, Linear Algebra and its Applications. New York: Academic, 1976.

[17] Y. Hua and T. Sarkar, "Matrix pencil method for estimating parameters of exponentially damped/undamped sinusoids in noise," IEEE Trans. Signal Process., vol. 38, no. 5, pp. 814-824, May 1990.
[18] A. Nehorai, "A minimal parameter adaptive notch filter with constrained poles and zeros," IEEE Trans. Acoust., Speech, Signal Process., vol ASSP-33, no. 8, pp. 983-996, Aug. 1985.

[19] P. Stoica and A. Nehorai, "Performance analysis of an adaptive notch filter with constrained poles and zeros," IEEE Trans. Acoust., Speech, Signal Process., vol. 36, no. 6, pp. 911-919, Jun. 1988.

[20] — "Convergence analysis of an adaptive pseudolinear-regression notch filtering algorithm," Circuits Syst. Signal Process., vol. 10, no. 2, pp. 245-259, 1991.

[21] P. Händel and A. Nehorai, "Tracking analysis of an adaptive notch filter with constrained poles and zeros," IEEE Trans. Signal Process., vol. 42 , no. 2, pp. 281-291, Feb. 1994.

[22] T. Kailath, Linear Systems. Englewood Cliffs, NJ: Prentice-Hall, 1980.

[23] M. Wax and I. Ziskind, "On unique localization of multiple sources by passive sensor arrays," IEEE Trans. Acoust., Speech, Signal Process., vol. 37, no. 7, pp. 996-1000, Jul. 1989.

[24] T. Laudadio, Y. Selén, L. Vanhamme, P. Stoica, P. Van Hecke, and S Van Huffel, "Subspace-based mrs data quantitation of multiplets using prior knowledge," J. Magn. Resonance, vol. 168, pp. 53-65, 2004.

[25] J. J. Fuchs, "Estimating the number of sinusoids in additive white noise," IEEE Trans. Acoust., Speech, Signal Process., vol. 36, no. 12, pp. 1846-1854, Dec. 1988.

[26] J.-K. Hwang and Y.-C. Chen, "A combined detection-estimation algorithm for the harmonic-retrieval problem," Signal Process., vol. 30, no 2, pp. 177-197, 1993.

[27] S. Kung, K. S. Arun, and D. V. B. Rao, "State space and singular value decomposition based approximation methods for the harmonic retrieval problem," J. Opt. Soc. Amer, vol. 73, pp. 1799-1811, 1983.

[28] M. Jansson and P. Stoica, "Forward-only and forward-backward sample covariances-A comparative study," Signal Process., vol. 77, pp. 235-245, 1999.

[29] T. Söderström, M. Sebek, J. Jezek, V. Kucera, and D. Henrion, “An evaluation of algorithms for computing the covariance function of a multivariable arma process," Eur. J. Control, vol. 8, no. 4, pp. 315-325, 2002.

[30] O. Besson and P. Stoica, "Analysis of MUSIC and ESPRIT frequency estimates for sinusoidal signals with lowpass envelopes," IEEE Trans. Signal Process., vol. 44, no. 9, pp. 2359-2364, Sep. 1996.

[31] _ "Estimation of the parameters of a random amplitude sinusoid by correlation fitting," IEEE Trans. Signal Process., vol. 44, no. 11, pp. 2911-2916, Nov. 1996.

[32] — "Two subspace-based methods for frequency estimation of sinusoidal signals with random amplitude," Proc. Inst. Elect. Eng. Radar Sonar Navig., vol. 144, pp. 169-176, 1997.

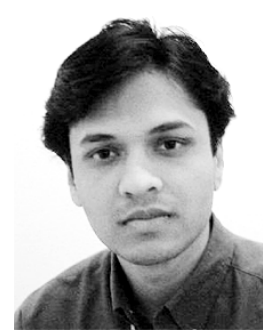

Kaushik Mahata received the M.E. degree in signal processing from the Indian Institute of Science, Bangalore, India, in 2000 and the Ph.D. degree in signal processing from Uppsala University, Uppsala, Sweden, in 2003. Currently, he is a research academic with the university of Newcastle, Callaghan, Australia. His research interest includes estimation identification and spectrum analysis. 